\title{
Human Transgene-Free Amniotic-Fluid-Derived Induced Pluripotent Stem Cells for Autologous Cell Therapy
}

\author{
Guihua Jiang, ${ }^{1,2}$ Julie Di Bernardo, ${ }^{2}$ Michael M. Maiden, ${ }^{2}$ Luis G. Villa-Diaz, ${ }^{3}$ Omar S. Mabrouk, ${ }^{4}$ \\ Paul H. Krebsbach, ${ }^{3}$ K. Sue O'Shea, ${ }^{1,5}$ and Shaun M. Kunisaki ${ }^{1,2,6}$
}

The establishment of a reliable prenatal source of autologous, transgene-free progenitor cells has enormous potential in the development of regenerative-medicine-based therapies for infants born with devastating birth defects. Here, we show that a largely CD117-negative population of human amniotic fluid mesenchymal stromal cells (AF-MSCs) obtained from fetuses with or without prenatally diagnosed anomalies are readily abundant and have limited baseline differentiation potential when compared with bone-marrow-derived MSCs and other somatic cell types. Nonetheless, the AF-MSCs could be easily reprogrammed into induced pluripotent stem cells (iPSCs) using nonintegrating Sendai viral vectors encoding for OCT4, SOX2, KLF4, and $\mathrm{cMYC}$. The iPSCs were virtually indistinguishable from human embryonic stem cells in multiple assays and could be used to generate a relatively homogeneous population of neural progenitors, expressing PAX6, SOX2, SOX3, Musashi-1, and PSA-NCAM, for potential use in neurologic diseases. Further, these neural progenitors showed engraftment potential in vivo and were capable of differentiating into mature neurons and astrocytes in vitro. This study demonstrates the usefulness of AF-MSCs as an excellent source for the generation of human transgene-free iPSCs ideally suited for autologous perinatal regenerative medicine applications.

\section{Introduction}

$\mathrm{P}$ ROGRESS IN THE FIELD of autologous tissue engineering and regenerative medicine continues to be hampered by a general lack of reliable and abundant sources of cells. The generation of patient-specific induced pluripotent stem cells (iPSCs) by the forced expression of defined, transgene-free reprogramming factors has the potential to provide an unlimited source of autologous cells, thereby leading to unique opportunities to develop novel, cellbased therapies for conditions that currently respond poorly to conventional treatment.

To date, iPSCs have been generated by nonintegrative techniques from numerous somatic cell types, including dermal fibroblasts, adipocytes, and mobilized CD34 ${ }^{+}$ cells. Unfortunately, none of these cell types are readily accessible for reprogramming for the fetus diagnosed with a major birth defect. Many of these patients-afflicted with a wide range of disorders, such as craniofacial malformations, myelomeningocele, congenital heart disease, congenital diaphragmatic hernia, pulmonary hypoplasia, and structural brain abnormalities-could substantially benefit from autologous iPSC-based technologies delivered in a timely fashion, either prenatally or in the early postnatal period.

Somatic cells normally present within amniotic fluid, the substance that surrounds the fetus throughout gestation, have been evaluated for decades as a diagnostic tool to rule out major karyotypic abnormalities in fetuses thought to be at increased risk for birth defects. However, only recently have these cells been explored for pluripotency and iPSC reprogramming capabilities [1-4]. Current knowledge suggests that multiple cell types exist, including mesenchymal stromal/stem cells (MSCs) and amniotic fluid stem (AFS) cells [5]. Given their potentially favorable cell reprogramming characteristics and relative ease of isolation across a wide range of gestational ages, we sought to generate iPSC lines derived from secondand third-trimester human amniotic-fluid-derived mesenchymal stromal cells (AF-MSCs) using nonintegrating, cytoplasmic Sendai virus $(\mathrm{SeV})$. These cells were subsequently compared with human embryonic stem cells (ESCs) and were selectively differentiated into various progenitor and mature neural derivatives.

\footnotetext{
${ }^{1}$ Pluripotent Stem Cell Lab, Departments of ${ }^{2}$ Surgery, ${ }^{3}$ Biologic and Materials Sciences, ${ }^{4}$ Chemistry, ${ }^{5}$ Cell and Developmental Biology, and ${ }^{6}$ Obstetrics and Gynecology, C.S. Mott Children's Hospital and Von Voigtlander Women's Hospital, University of Michigan Medical School, Ann Arbor, Michigan.
} 


\section{Materials and Methods}

\section{Isolation of human AF-MSCS}

Approval for this work was obtained from the Institutional Review Board and the Human Pluripotent Stem Cell Research Oversight Committee at the University of Michigan (No. 38565). After written informed consent, secondand third-trimester human amniotic fluid samples $(n=7$, range $=18-37$ weeks of gestation) were retrieved during amniocentesis or fetoscopic intervention from pregnancies with or without fetal anomalies detected by ultrasound at Von Voigtlander Women's Hospital, University of Michigan Health System. In addition to healthy controls $(n=2)$, fetal anomalies included twin-twin transfusion syndrome (TTTS, $n=2)$, cleft lip/palate $(n=1)$, congenital heart disease $(n=1)$, and congenital diaphragmatic hernia $(n=1)$. In each case, a normal fetal karyotype was confirmed by our clinical laboratory within 2 weeks after specimen procurement. Cells were washed twice in PBS prior to resuspension in mesenchymal basal medium [DMEM, 20\% fetal bovine serum (FBS), $100 \mathrm{U} / \mathrm{mL}$ penicillin and $100 \mu \mathrm{g} / \mathrm{mL}$ streptomycin, and $2 \mathrm{mM}$ L-glutamine (Invitrogen)] and incubated at $37^{\circ} \mathrm{C}$ with $5 \%$ humidified $\mathrm{CO}_{2}$. After $24-48 \mathrm{~h}$, nonadherent cells were removed by washing the plates with phosphatebuffered saline, and the medium was replaced as previously described [6]. Upon reaching $75 \%$ confluence, cells were detached using Trypsin (Invitrogen) and seeded at a split ratio of 1:4.

\section{iPSC derivation}

Two days before transduction, unsorted $2 \times 10^{5}$ AF-MSCs isolated from four patients selected at random were placed into six-well plates. Integration-free iPSC derivation was performed on AF-MSCs using $\mathrm{SeV}$ vectors encoding for OCT4, SOX2, KLF4, and cMYC (OSKM) at $3 \times 10^{6} \mathrm{CIU}$ (CytoTune $^{\mathrm{TM}}$-iPS Reprogramming Kit; Invitrogen) as previously described elsewhere for postnatal dermal fibroblasts $[7,8] . \mathrm{SeV}$ is a negative sense, single-stranded RNA virus that does not integrate into the host genome and replicates exclusively in the cytoplasm. AF-MSCs reprogrammed using conventional four Yamanaka factor retrovirus were used as controls. Twenty-four hours later, the medium was replaced with mesenchymal basal medium. At day 6 , the cells were split and placed on irradiated mouse embryonic fibroblast (MEF) feeder layers. All cells, including H9-ESC controls (WiCell), were cultured in human ES conditioned medium (GlobalStem) with $4 \mathrm{ng} / \mathrm{mL}$ of FGF-2 (Millipore). Individual colonies derived from MSCs (AF-iPSC-SeV) were subsequently picked and propagated on MEFs, Matrigel (BD Biosciences), or PMEDSAH, a defined synthetic matrix polymer [9]. Alkaline phosphatase staining was performed using the AP substrate kit (Millipore) according to manufacturer's instructions.

\section{Directed neural differentiation}

Differentiation of iPSCs into neural progenitors was conducted based on modifications of a protocol described elsewhere [10]. Briefly, three different AF-iPSC-SeV lines at passage 7 or 8 were manually cut and transferred into 60$\mathrm{mm}$ petri dishes for 4 days in human ES conditioned me- dium without FGF-2 followed by culture in neural induction medium (DMEM/F12, GlutaMAX, NEAA, and N2; Invitrogen) for 2 days. The resulting neurospheres were transferred into polyornithine/laminin-coated six-well plates (Sigma-Aldrich) to facilitate rosette formation. Individual rosettes were manually isolated, dissected, and replated into dishes containing neural stem cell media [Neurobasal media, B27 (Invitrogen), NEAA, GlutaMAX, and FGF-2]. Differentiation of neural progenitors into neurons was performed by culturing neural progenitors in Neurobasal media, B27, and GlutaMAX on poly-L-ornithine- and laminin (Sigma-Aldrich)-coated 12-well plates for up to 70 days. Astrocyte differentiation was induced by culturing neural progenitors in DMEM, N2, GlutaMAX, and 1\% FBS for 4 weeks.

\section{Flow cytometry}

AF-MSCs between passage 3 and 5 were evaluated for MSC characteristics based on criteria as described elsewhere [11]. For flow cytometry, primary antibodies used were phycoerythron (PE)-conjugated against CD34, CD44, CD45, CD73, CD90, CD105, CD117, OCT4A, SOX2, SSEA3, SSEA4, TRA-1-60, TRA-1-81, and HLA-DR (all from BD Biosciences). Cells were fixed with $2 \%$ paraformaldehyde prior to evaluation of antibody staining using the LSRII flow cytometer (BD Biosciences). Resultant data were analyzed using FloJo software (Tree Star).

\section{Colony-forming units}

To evaluate the proliferation and clonogenic capacity of MSCs in vitro, colony-forming unit-fibroblast (CFU-F) assays were performed in triplicate as previously described [8]. Cells were seeded in serial dilution in 96-well plates starting from an initial inoculum of $10^{3}$ cells per well and cultured in mesenchymal basal medium for 10 days, fixed with $10 \%$ buffered paraformaldehyde, and stained with a $0.1 \%$ solution of crystal violet. The CFU-F was determined by the number of colonies ( $\geq 20$ cells/colony) per 100 cells seeded.

\section{MSC differentiation}

Adipogenic, osteogenic, and chondrogenic differentiation of MSCs was performed at passage 4 using the following protocols [12]. For adipocyte differentiation, confluent cells were placed in adipogenic differentiation medium $[0.5 \mathrm{mM}$ isobutylmethylxanthine (EMD Millipore), $200 \mu \mathrm{M}$ indomethacin (MP Biomedicals), $10^{-6} \mathrm{M}$ dexamethasone (MP Biomedicals), and $10 \mu \mathrm{g} / \mathrm{mL}$ of insulin (Sigma-Aldrich) in aMEM (Invitrogen)] supplemented with $10 \%$ FBS. After 21 days, control and treated cells were fixed with $10 \%$ buffered paraformaldehyde and intracellular lipid droplets were stained with $0.5 \%$ oil red O (Invitrogen) or using an antibody against fatty acid binding protein-4 (FABP4; R\&D Systems). For osteoblast differentiation, confluent cells were cultured in osteogenic differentiation medium $\left[10^{-7} \mathrm{M}\right.$ dexamethasone (MP Biomedicals) and $10 \mathrm{mM}$ $\beta$-glycerophosphate (Alfa Aesar) in DMEM] supplemented with $10 \%$ FBS. After 21 days, cells were fixed as earlier and assessed for matrix mineralization by $0.6 \%$ alizarin red $\mathrm{S}$ staining or an antibody against osteocalcin (OC; R\&D 
Systems). For chondrocyte differentiation, cells were cultured as micromass pellets in differentiation medium (DMEM, 10 ng/ mL TGF- $\beta$, and $10^{-7} \mathrm{M}$ dexamethasone) supplemented with $10 \%$ FBS. After 28 days, pellets were fixed with $10 \%$ buffered paraformaldehyde, embedded in OCT compound (TissueTek), and hexane cooled over an acetone dry-ice slurry. Ten-micrometer-thick cryosections were stained with a $1 \%$ solution of alcian blue or an antibody against aggrecan (R\&D Systems).

\section{Embryoid body assay}

Embryoid bodies (EBs) were formed in suspension culture and analyzed using methods previously described [13]. Briefly, AF-iPSC-SeV colonies derived from each parental AF-MSC were transferred into low-adherence dishes containing human ES conditioned medium. After 14 days in suspension culture, EBs were placed in 12-well plates for up to 28 days to allow spontaneous differentiation prior to gene expression and immunocytochemical analysis.

\section{Reverse transcription-polymerase chain reaction and quantitative polymerase chain reaction}

Total RNA was extracted from cells using TRIzol (Invitrogen). First-strand cDNA was prepared with the Taqman Reverse Transcription Reagent kit (Applied Biosciences). Reverse transcription (RT)-polymerase chain reaction (PCR) was performed in triplicate at 35 cycles (Life Technologies). The primer sequences for RT-PCR can be found in Supplementary Table S1 (Supplementary Data are available online at www.liebertpub.com/scd). Quantitative PCR (qPCR) was carried out on a Mastercycler ep realplex (Eppendorf) using Fast SYBR Green Master Mix (Applied Biosystems) as recommended by manufacturer. Relative gene expression was analyzed by qPCR with GAPDH used as a reference gene to normalize target gene expression using the $2^{-\Delta \Delta \mathrm{Ct}}$ method [14]. The primer sequences used for qPCR are provided in Supplementary Table S2.

\section{Global gene expression analysis}

To compare transcriptomes of parental cell types and pluripotent stem cells, microarray analysis was performed. Total RNA was extracted using TRIzol from one randomly selected, Sendai-virus-reprogrammed iPSC clone and its parental AFMSC line at three different passages (range $=5-15$ ). Total RNA from dermal fibroblasts and H9-ESCs from a single passage were used as controls. Sample processing, probing to the Human Gene 2.1 ST array platform (Affymetrix), and data analysis were performed by the University of Michigan DNA Sequencing Core Microarray Facility. Normalized expression by robust multiple-array averages was plotted using the heatmap.2 function from the gplots package in R (www.rproject.org) using default parameters. The euclidean distance dissimilarity matrix and complete linkage method were used to generate the dendrogram. Expression values range from $\sim 1$ to 12 with lower expression values shown in red and higher expression values shown in blue.

\section{Immunocytochemical analyses}

To assay pluripotency in each established AF-iPSC-SeV cell line, primary antibodies against NANOG (Abcam),
OCT4 (Santa Cruz), SOX2, SSEA3, SSEA4, TRA-1-60, and TRA-1-81 (Millipore) were used following fixation in $4 \%$ paraformaldehyde. Primary antibodies used to evaluate differentiation were against Nestin (Millipore), Neuron-specific class $\beta$-III tubulin (TuJ1; Covance), PSA-NCAM (Millipore), $\alpha$-fetoprotein (AFP), FOXA2, and SOX17 (R\&D Systems). Cells were examined using epifluorescence microscopy (DM IRB; Leica).

\section{Cytogenetics}

Karyotyping was performed on AF-iPSC-SeVs and neural progenitors derived from AF-iPSC-SeVs (AF-NPC-SeVs) every five passages by Cell Line Genetics. Chromosome spreads were prepared using standard protocols and measurements were performed using the GTL-banding method on 20 metaphase preparations.

\section{Neurosurgical implantation and analysis}

Cell transplantation in a neonatal rodent model was conducted with approval of the University of Michigan Committee on the Use and Care of Animals (No. 10447-1). AF-NPC-SeVs were incubated with magnetic microbeads (Miltenyi Biotec) conjugated with PSA-NCAM antibody at $4^{\circ} \mathrm{C}$ for $10 \mathrm{~min}$ and positively selected by cell sorting. Oneday-old Sprague-Dawley rat pups $(n=4)$ were anesthetized with isoflurane. PSA-NCAM-positive cells were injected (250,000 cells/injection in $5-\mu \mathrm{L}$ volume of PBS) through the sagittal suture directly into the brain parenchyma using a stereotactic frame (David Kopf Instruments). The incision was closed with 4-0 monocryl (Ethicon) suture. Rats were euthanized at either 1 or 6 weeks after implantation. For immunohistochemical analysis, all animals were transcardially perfused with $10 \%$ formalin. The brains were collected and postfixed overnight prior to cryoprotection in 30\% sucrose solution. Brains were embedded in OCT compound and hexane cooled over an acetone dry-ice slurry. Eighteenmicrometer-thick coronal cryosections were cut and probed with a mouse anti-human nuclear antigen (HNA) antibody (Millipore). Stained sections were mounted using Prolong Gold Antifade Reagent with DAPI (Life Technologies) and analyzed with a confocal microscope (Nikon).

\section{Statistical analyses}

Quantitative data analyses were performed in triplicate and presented as the mean \pm SEM. The data were analyzed by the Student's $t$-test or analysis of variance with Bonferroni correction for multiple comparisons using GraphPad Prism software (version 5.0d). Results were considered to be statistically significant if $P \leq 0.05$ unless otherwise stated.

\section{Results}

\section{Human AF-MSCs have unique phenotypic characteristics and limited mesenchymal differentiation potential}

We isolated AF-MSCs without cell sorting from secondand third-trimester amniotic fluid from fetuses with and without prenatally diagnosed congenital anomalies. Within 1 week after seeding in mesenchymal basal medium, plastic 
adherent, fibroblast-appearing cells were detected in the majority $(5 / 7,71.4 \%)$ of amniotic fluid samples across a wide range of gestational ages (mean $=22.5$ weeks). The AF-MSCs grew in monolayers and displayed a relatively homogeneous, spindle-shaped morphology after the first passage (Fig. 1A, upper left). Their fetal origin and lack of maternal contamination among AF-MSCs harvested from male fetuses was confirmed by SRY and X-linked amelogenin qPCR assays (Fig. 1B). The AF-MSCs were clonogenic, demonstrating a CFU-F of 0.8 colonies for every 100 cells seeded (Fig. 1A, lower left). AF-MSCs were successfully maintained in continuous culture for over 3 months (passage 8) without morphologic change or evidence of reduced cell proliferation.

To evaluate the mesodermal differentiation potential of AFMSCs, directed differentiation experiments were performed and confirmed that a subset of AF-MSCs were capable of differentiating into different mesodermal cell types, namely, adipocytes and osteoblasts, when cultured in the appropriate directive media for 3 weeks (Fig. 1A). Evidence of adipogenic differentiation was indicated by the presence of oil red $\mathrm{O}$ positive lipid vacuoles within the cytoplasm of a subpopulation of AF-MSCs (Fig. 1A, upper middle) and by expression of FABP4 (Fig. 1A, lower middle) by immunofluorescence. There was minimal osteogenic differentiation in only a subset of AF-MSCs as evidenced by increased extracellular calcium matrix staining in the presence of alizarin red (Fig. 1A, upper right) and by positive expression of the late osteogenic marker OC (Fig. 1A, lower right). However, the degree of adipogenic and osteogenic differentiation was far less robust in AF-MSCs compared with that observed with unsorted human bonemarrow-derived MSCs (data not shown). Further, there was no evidence of chondrocyte differentiation among AF-MSCs cultured as micromass pellets under chondrogenic conditions.

Given the apparent reduced differentiation potential of AF-MSCs and their morphologic similarities to dermal

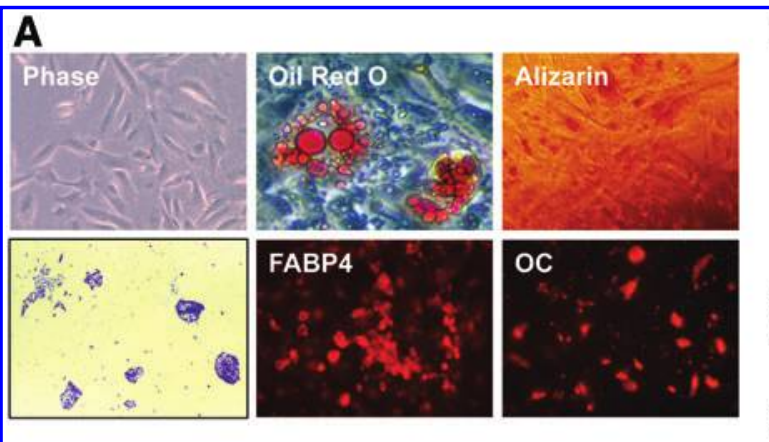

D

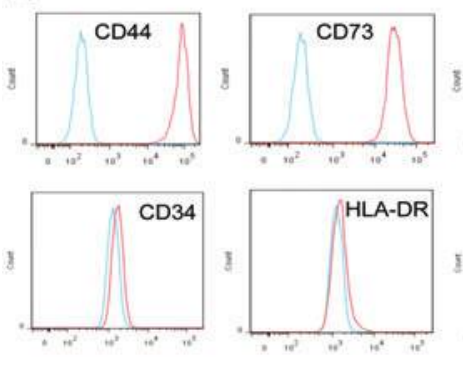

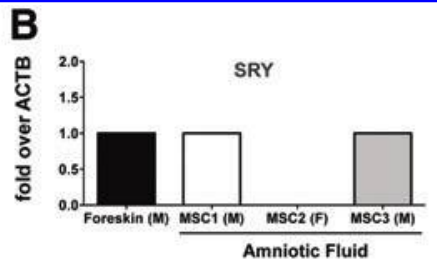
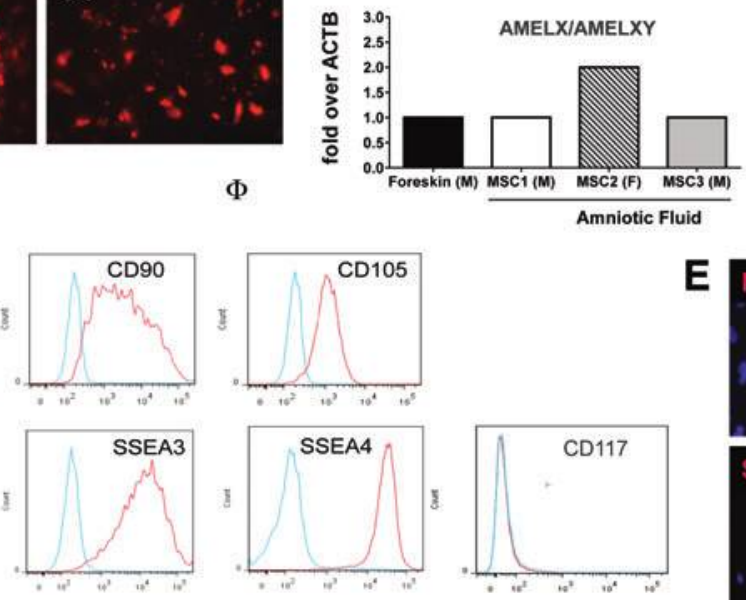
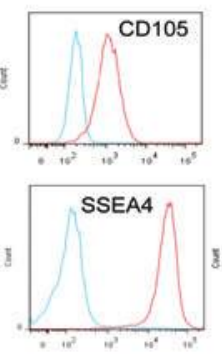

C

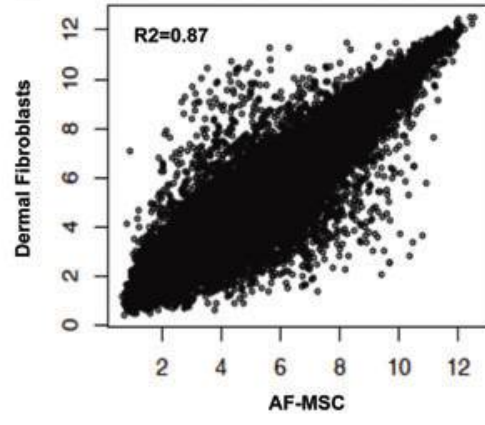

E
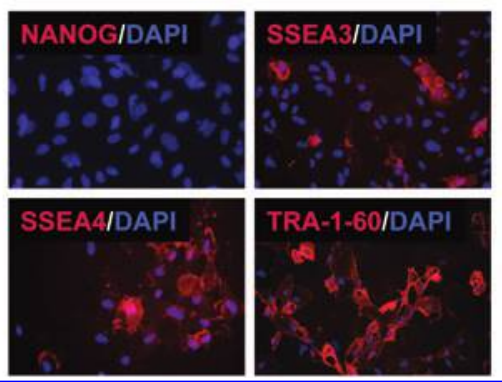

FIG. 1. Characterization of human amniotic fluid mesenchymal stromal cells (AF-MSCs). (A) Phase-contrast photomicrograph illustrating typical AF-MSC morphology at passage 4 (upper left, magnification, $100 \times$ ). Representative colony forming unit-fibroblast (CFU-F) from AF-MSCs stained with $0.1 \%$ crystal violet (lower left, magnification, $5 \times$ ). Oil red $\mathrm{O}$ staining of AF-MSCs grown under adipogenic conditions for 21 days reveals orange-red cytoplasmic lipid droplets consistent with adipocytes (upper middle, magnification, $100 \times$ ). Immunofluorescence microscopy of AF-MSCs under adipogenic conditions demonstrates expression of adipogenic marker, fatty acid binding protein 4 (FABP4, lower middle, magnification, 100 $\times$ ). Alizarin red S staining of AF-MSCs in osteogenic conditions for 21 days shows extracellular mineral deposition (upper right, magnification, $100 \times)$. Immunofluorescence microscopy of AF-MSCs under osteogenic conditions demonstrates expression of osteogenic marker, osteocalcin (OC, lower right, magnification, 100×). (B) SRY (upper) and amelogenin (lower) gene expression among isolated AF-MSCs by representative quantitative polymerase chain reaction (qPCR). Absence of SRY in a female fetal sample (MSC2) and upregulation of SRY in male fetal samples (MSC1 and MSC3) comparable to foreskin dermalfibroblast-positive control. Upregulation of X-linked amelogenin (AMELX) in a female fetal sample (MSC2) compared with male fetal samples (MSC1 and MSC3) and foreskin dermal fibroblasts. (C) Global gene expression patterns of AF-MSCs were compared with those of neonatal dermal fibroblasts analyzed using regression analyses. $R^{2}$ denotes the coefficient of determination. (D) Flow cytometry characterization of AF-MSCs (positive shown in red compared with blue negative control) at passage 4 grown in mesenchymal basal media, suggesting a unique immunophenotypic profile with positive staining for MSC markers (CD44, CD73, CD90, and CD105) and selected pluripotency markers (SSEA3 and SSEA4). AF-MSCs do not stain for the hematopoietic marker (CD34), CD117, or the class II major histocompatibility complex antigen HLA-DR. (E) Immunofluorescence microscopy of AF-MSCs merged with DAPI (in blue) showing lack of NANOG expression but expression of SSEA3, SSEA4, and TRA-1-60 (magnification, 100×). Color images available online at www.liebertpub.com/scd 
fibroblasts, we next sought to determine whether AF-MSCs were similar to fully differentiated dermal fibroblasts, the most common postnatal parental cell type used for reprogramming toward pluripotency. Using a microarray representing the genome-wide transcriptome and regression analysis, the global gene expression profile of primary AFMSCs was different from that of the neonatal dermal fibroblast cell line $\left(R^{2}=0.87\right.$, Fig. $\left.1 C\right)$.

The immunophenotypic profile of unsorted AF-MSCs was further characterized by flow cytometry. AF-MSCs uniformly expressed surface epitope markers associated with bone-marrow-derived MSCs (Fig. 1D), including CD44 (mean $=96.7 \%$ of cells), CD73 (95.7\%), CD90 (88.9\%), and CD105 (83.4\%). In contrast, less than one percent of cells expressed HLA-DR or hematopoietic stem cell markers, including CD34 and CD45 (data not shown). Although the pluripotency marker NANOG was not detected (data not shown), $62.8 \%$ and $66.8 \%$ of cells displayed SSEA3 and SSEA4, respectively (Fig. 1D). The percentage of AF-MSCs that expressed CD117 was either very low or undetectable in all samples $($ mean $=0.66 \%)$. To further support the unique, mixed phenotypic signature of AF-MSCs, we performed immunofluorescence staining, which showed lack of NANOG expression but positive expression of SSEA3 and SSEA4, and in some established primary AF-MSCs, TRA-160 (Fig. 1E).

\section{AF-MSCs can be readily reprogrammed into transgene-free pluripotent cells}

Successful OSKM reprogramming of unsorted AF-MSCs $(n=4)$ with the nonintegrating $\mathrm{SeV}$ was observed when cultured under standard ESC conditions. AF-MSCs began to aggregate while reducing cytoplasmic volume and losing their spindle-shaped morphology between 14 and 21 days in culture (Fig. 2A, left). At 28 days, there were multiplecandidate iPSC colonies with well-defined borders and high nuclear-to-cytoplasm ratios analogous to undifferentiated H9-ESCs. We confirmed that AF-iPSC-SeVs expressed high levels of alkaline phosphatase (Fig. 2A, right). Using

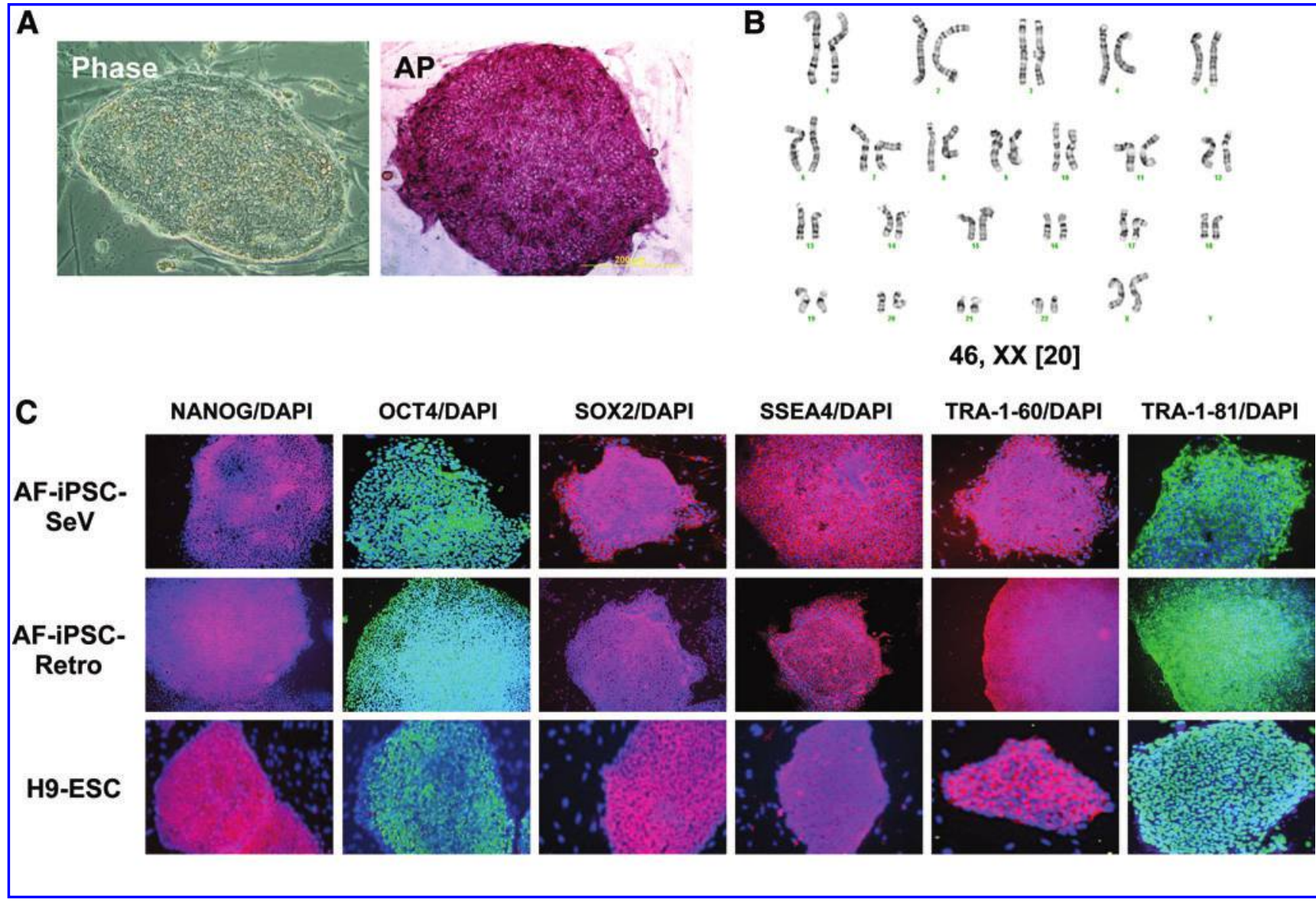

FIG. 2. Characterization of human induced pluripotent stem cells (iPSCs) derived from amniotic fluid (AF) mesenchymal stromal cells. (A) Representative colony morphology of AF-iPSCs using nonintegrating Sendai virus (SeV) grown on mouse embryonic feeders at day 18 (left, magnification, $100 \times$ ). Large AF-iPSC-SeV colony with evidence of pluripotency by alkaline phosphatase (AP) staining (right, magnification, 20×). (B) Representative karyotype analysis of AF-iPSC-SeV colonies based on $20 \mathrm{G}$-banded metaphase cells demonstrating normal chromosomes (passage 18). (C) Immunofluorescence photomicrographs of AF cells reprogrammed with $\mathrm{SeV}$ (AF-iPSC-SeV, upper), AF cells reprogrammed with retrovirus (AFiPSC-Retro, middle), and H9-ESC controls (lower) on mouse feeder layers, demonstrating expression of the following pluripotency markers: NANOG, OCT4, SOX2, SSEA3, TRA-1-60, and TRA-1-81 (magnification, 20×). DAPI staining in blue. Color images available online at www.liebertpub.com/scd 
OSKM SeV, the overall reprogramming efficiency of AFMSCs into iPSCs ranged from $0.01 \%$ to $0.05 \%$ and was comparable to the efficiency seen using AF-MSCs with OSKM retrovirus (AF-iPSC-Retro).

A total of 10 different AF-iPSC-SeV lines were successfully transferred onto feeder-free systems, including either Matrigel- or PMEDSAH-coated dishes, for further expansion, subculture, and additional characterization. There were no major differences in morphology based on these different culture conditions. Cytogenetic analyses showed normal karyotypes, indicating that AF-iPSC-SeVs remained free of nonclonal aberrations after continuous in vitro expansion to passage 20 (Fig. 2B). Immunofluorescence staining revealed uniformly high expression of NANOG, OCT4, SOX2, SSEA3, SSEA4, TRA-1-60, and TRA-1-81 within AF-iPSC-SeV colonies in a pattern similar to that seen with AF-iPSC-Retro and H9-ESC colonies (Fig. 2C).

Qualitative gene expression analyses of different AFiPSC-SeV clones demonstrated upregulation of key pluripotency genes, including NANOG and SOX2, compared with low levels observed with parental primary AF-MSCs
(Fig. 3A). There was endogenous expression of OCT4 among all AF-MSCs that were maintained following reprogramming. Loss of $\alpha$-smooth muscle actin ( $\alpha$-SMA) amplification normally present in AF-MSCs was seen following conversion to AF-iPSC-SeVs (data not shown). Evidence for genomic integration of $\mathrm{SeV}$ vectors was evaluated by RT-PCR. From passage 6 onward, the SeV transgene was nearly undetectable but strong expression of NANOG among AF-iPSC-SeV clones remained (Fig. 3B). Complete absence of $\mathrm{SeV}$ transgene expression in the presence of NANOG amplification was noted by passage 10 .

In accordance with the RT-PCR results, quantitative gene expression studies of AF-iPSC-SeVs showed significant upregulation in NANOG compared with parental AF-MSCs $(P \leq 0.05$, Fig. 3C). There was a similar significant increase in SOX2 and KLF4 among AF-iPSC-SeVs comparable to levels observed in H9-ESCs. Despite high endogenous expression of OCT4 among AF-MSCs shown by RT-PCR, qPCR revealed significant increases in OCT4 transcripts after $\mathrm{SeV}$ reprogramming when normalized for baseline GAPDH expression $(P \leq 0.05)$. There was a significant decrease in cMYC amplification in AF-iPSC-SeVs compared

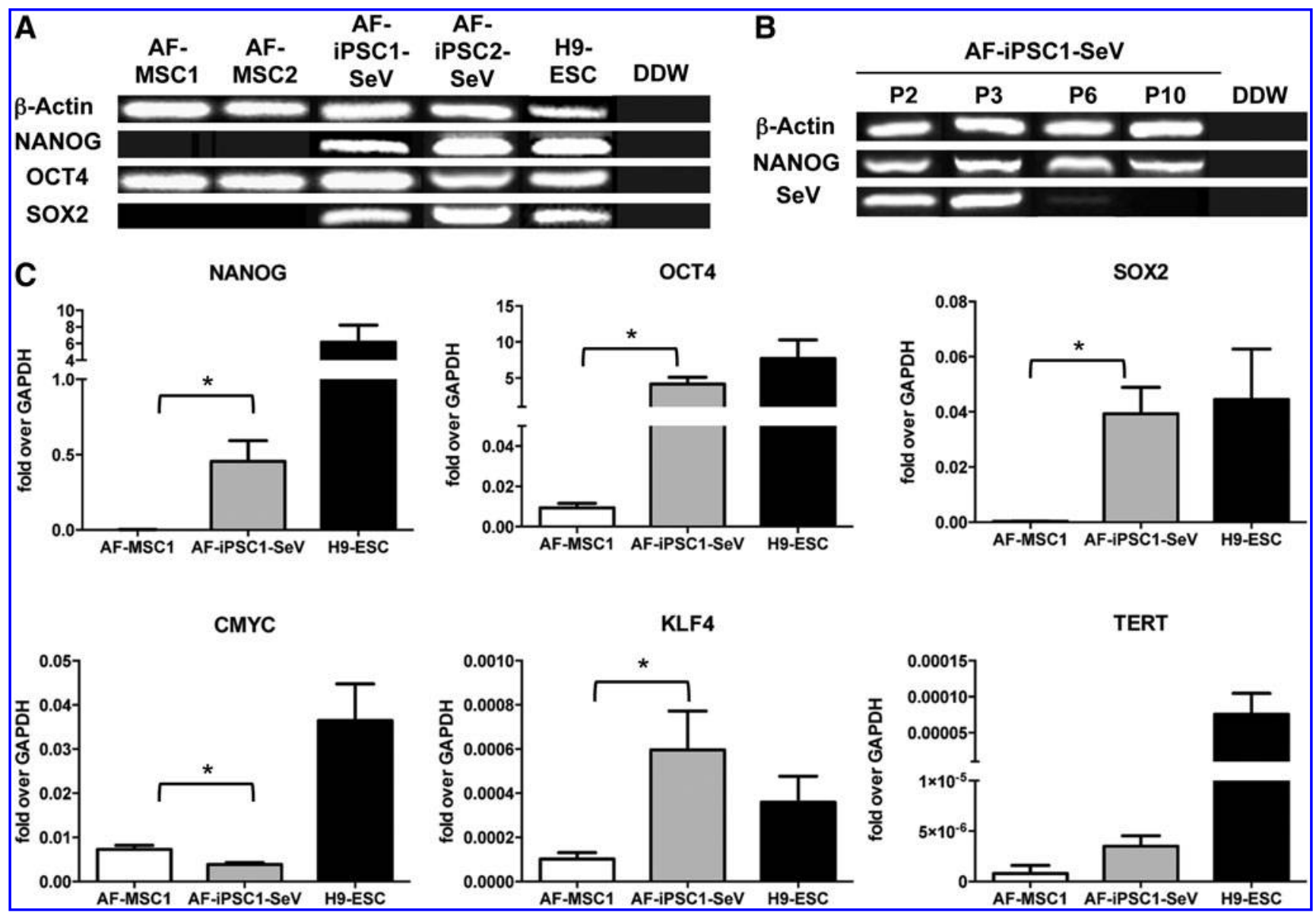

FIG. 3. Gene expression of representative human amniotic-fluid-derived iPSCs reprogrammed with nonintegrating Sendai virus. (A) Reverse transcription (RT)-PCR of iPSC RNAs showing maintenance of OCT4 and upregulation of NANOG and SOX2. (B) Representative RT-PCR from an AF-iPSC-SeV clone demonstrating maintenance of NANOG but loss of the SeV transgene sequences by passage 6 (P6). (C) Quantitative analysis by qPCR demonstrating marked upregulation of selected pluripotency genes, including NANOG, OCT4, SOX2, and KLF4, among AF-iPSC-SeVs $(n=3)$ to levels comparable to that of H9-ESCs. Data were normalized relative to dermal fibroblast controls and presented as the mean $\pm \mathrm{SEM}(n=5, * P \leq 0.05)$. 
with AF-MSCs $(P \leq 0.05)$, but no significant difference in TERT expression.

\section{Global gene expression of AF-iPSC-SeVs is similar to that of ESCS}

To determine the similarities and differences of $\mathrm{AF}$ iPSC-SeVs versus ESCs, we compared an AF-iPSC-SeV clone at three different passages to parental AF-MSCs and H9-ESCs in a microarray representing the genome-wide transcriptome. The dendrogram of one-way unsupervised hierarchical clustering analysis demonstrated that AFiPSC-SeV clones clustered with H9-ESCs, distinct from a cluster containing parental AF-MSCs and dermal fibroblasts (Fig. 4A). To assess the similarities and differences between the various cell types in more detail, a heatmap of 5,285 upregulated and downregulated genes was generated, which revealed transcriptome patterns in AF-iPSC-SeVs that were nearly identical to H9-ESCs (Fig. 4B). In accordance with the qPCR data, focused analysis of key pluripotency genes, including NANOG, OCT4, SOX2, LIN28, DPPA2, and FGF4, showed no significant differences between AF-iPSC-SeVs and H9-ESCs. In contrast, with the exception of KLF4, there was significant upregulation of pluripotency-associated genes in AF-iPSC-SeV clones when compared with primary AF-MSCs (data not shown, $P<0.001)$. Finally, global gene expression patterns of AF-iPSC-SeVs were nearly identical to those of H9ESCs $\left(R^{2}=0.97\right)$ and divergent from those of parental AFMSCs $\left(R^{2}=0.86\right.$, Fig. 4 C).

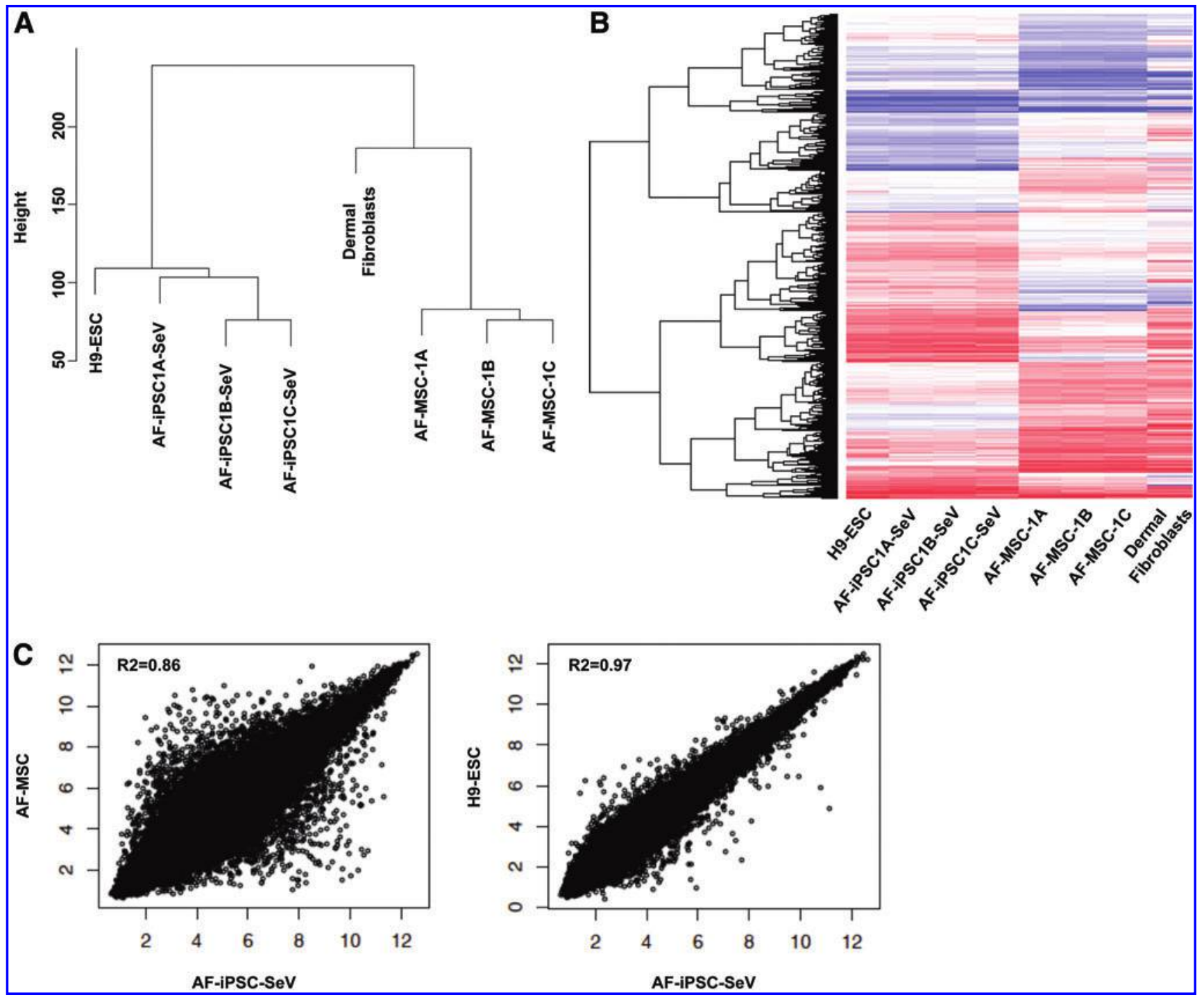

FIG. 4. Global gene expression analysis of amniotic-fluid-derived iPSCs using Sendai virus. (A) Dendrogram shows the unsupervised hierarchal clustering of AF-iPSC-SeVs, and parental AF-MSCs (both analyzed at three different passages) compared with dermal fibroblast and H9-ESC controls. (B) Heatmap reveals the relationship of 5,285 genes differentially expressed by H9-ESCs, AF-iPSC-SeVs, AF-MSCs, and dermal fibroblasts. Higher and lower levels of transcripts are shown in blue and red, respectively. The transcriptome patterns expressed by AF-iPSC-SeVs were nearly identical to those expressed by H9-ESCs (far left). (C) Global gene expression patterns of AF-iPSC-SeVs were more closely correlated with patterns observed in H9-ESCs (right) compared with those from parental primary cell lines (AF-MSCs, left). $R^{2}$ denotes the coefficient of determination. Color images available online at www.liebertpub.com/scd 


\section{AF-iPSC-SeVs are capable of spontaneous three-germ layer differentiation in vitro}

In an effort to determine whether AF-iPSC-SeVs were pluripotent in vitro, iPSC clones derived from three different AF-MSC samples were allowed to spontaneously differentiate in low-adherence cultures as EBs. Whereas AF-MSCs had neither the ability to form EBs nor the capability of surviving in suspension culture, AF-iPSC-SeVs exhibited robust EB formation after 7 days in suspension culture. Between 21 and 28 days, cells attached and adopted a flattened morphology at the periphery. Further, immunocytochemical analysis of AF-iPSC-SeV-EBs confirmed the presence of derivatives of three germ layers similar to EBs generated from retroviral-reprogrammed AF-MSCs (Fig. 5A). AF-iPSC-SeV-EBs expressed for endoderm markers, including AFP, SOX17, and FOXA2 (Fig. 5A, right). Mesodermal differentiation was confirmed by the presence of $\alpha$-SMA, and ectodermal differentiation was shown by positive Nestin and TuJ1 expression. Similarly, RT-PCR analyses of RNAs from EBs derived from AF-iPSCs-SeVs demonstrated successful differentiation into endodermal cells by AFP and GATA4 expression, into mesodermal cells by $\alpha$-SMA, DCN, and RUNX1 expression, and into ectodermal cells by Nestin and TuJ1 expression (Fig. 5B).

\section{SeV-reprogrammed AF-MSCs can differentiate into neural progenitors}

To test whether transcription-factor-free, amniotic-fluidderived iPSCs could be differentiated into early neural lineages, AF-iPSC-SeVs at passage 8 were exposed to neural differentiation culture conditions. Neurospheres attached and formed neural rosettes typical of neuroepithelial cells within 7 days of adherent culture (Fig. 6A, left). These neural progenitor colonies were subsequently detached, expanded, and analyzed at passage 8 . In this neurogenic environment, these morphologically distinct cells no longer expressed NANOG and OCT4 by immunofluorescence (Fig.
6A). Further, they expressed multiple early neural markers, including SOX2, SOX3, PAX6, Nestin, MSI1, and PSANCAM (Fig. 6B). With the exception of SOX2, undifferentiated AF-iPSC-SeVs did not express these neural progenitor markers (data not shown). Evaluation of cell surface marker epitopes by flow cytometry confirmed the increased expression of PSA-NCAM and decreased expression of SSEA4 following directed differentiation of AF-iPSC$\mathrm{SeVs}$ into the early neural lineage (Fig. 6C). The mean percentage of PSA-NCAM-positive cells increased from $3 \%$ to 53\% whereas SSEA3- and SSEA4-positive cells decreased to $0.8 \%$ and $0.1 \%$, respectively. RT-PCR analyses of neural progenitor cells differentiated from AF-NPC-SeVs downregulated NANOG and OCT4 and upregulated the neural progenitor transcription factors SOX1 and PAX6 (Fig. 6D). Karyotype analysis of AF-NPC-SeVs performed between passage 5 and 8 demonstrated normal chromosomes based on 20 G-banded metaphase cells. The only nonclonal aberration detected was trisomy 12 , an abnormality that is generally identified at a frequency of $2.5 \%$.

To confirm the ability of human AF-NPC-SeVs to successfully engraft into the central nervous system in vivo, PSA-NCAM-positive cells were injected into the cerebrum of neonatal rats. Despite xenotransplantation of donor cells into an immunocompetent host, histologic analyses showed survival of the AF-NPC-SeVs when examined 8 days after injection. An abundant signal of HNA-positive cells was readily identified adjacent to the delivery site in both animals but not in the adjacent native brain parenchyma (Fig. 6E). Six weeks after injection, there was no convincing evidence of HNA-positive cells in evaluated animals (data not shown).

\section{Neural progenitors can differentiate into neuronal and glial progeny in vitro}

To verify the multilineage differentiation capacity of AFNPC-SeVs, we performed directed in vitro experiments

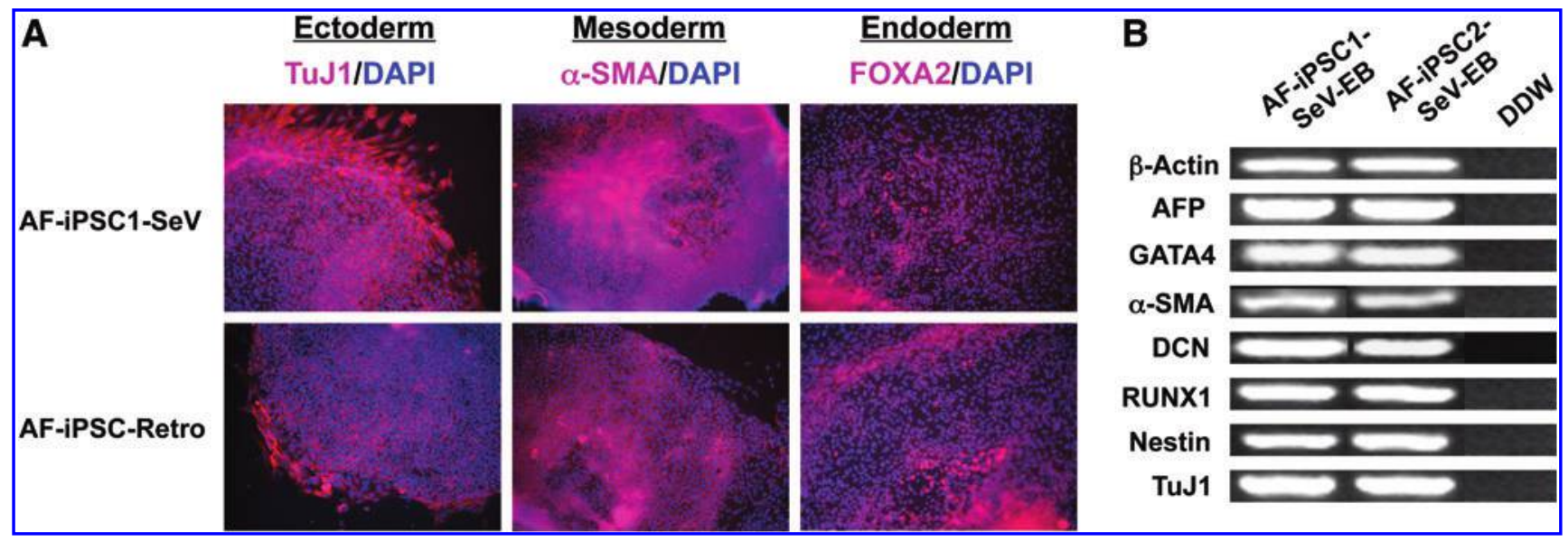

FIG. 5. Representative embryoid body (EB) formation of amniotic-fluid-derived iPSCs using Sendai virus. (A) Immunofluorescence microscopy of EBs from iPSCs reprogrammed with Sendai virus (AF-iPSC1-SeV) and retrovirus (AFiPSC-Retro) merged with DAPI (in blue) showing expression of TuJ1 (ectoderm), $\alpha$-smooth muscle actin ( $\alpha$-SMA) (mesoderm), and FOXA2 (endoderm, magnification, 20×). Similar three germ layer expression was also seen among EBs from H9-ESCs (data not shown). (B) RT-PCR from AF-iPSC-SeV-EB RNA extracted at 28 days, demonstrating activation of endodermal, mesodermal, and ectodermal genes. Color images available online at www.liebertpub.com/scd 
A
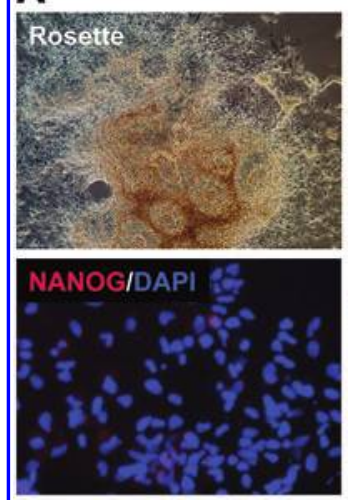

C

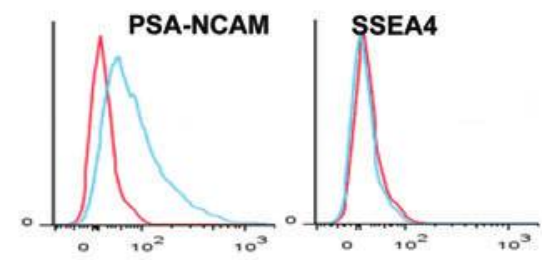

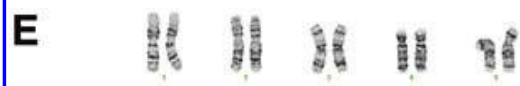

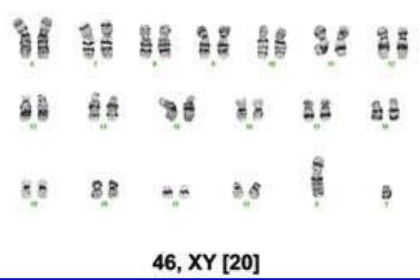

B
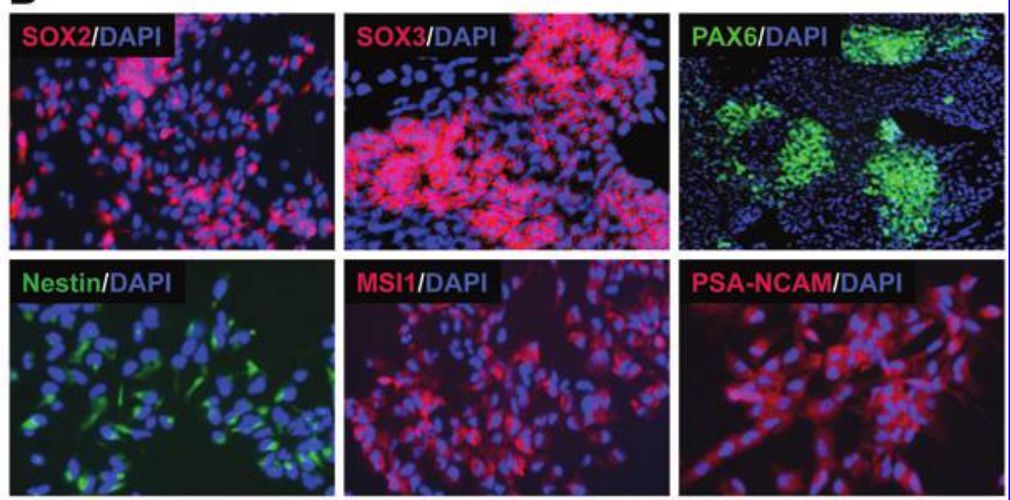

D

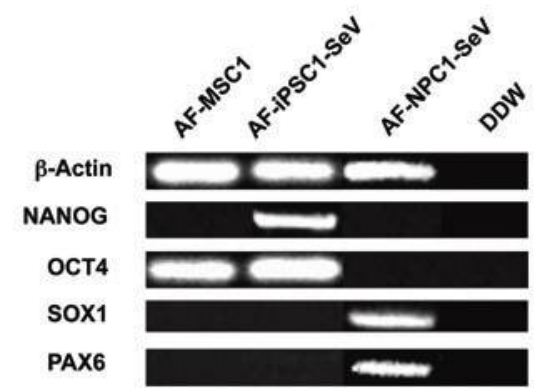

$\mathbf{F}$
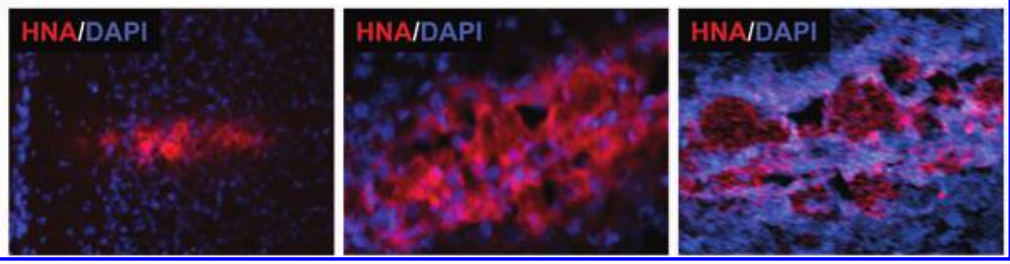

FIG. 6. Directed differentiation of AF-iPSC-SeVs into neural progenitor cells (AF-NPC-SeV) in vitro. (A) Photomicrograph of typical neural rosette formation under phase-contrast microscopy (upper left, magnification, 20 $\times$ ), and morphology of representative neural stem cells at several passages under phase microscopy (NSCs) (upper right, magnification, $100 \times$ ). Merged photomicrographs showing lack of expression of two pluripotency markers, NANOG (lower left, magnification, $100 \times$ ) and OCT4 (lower right, magnification, 40×). DAPI in blue. (B) Merged photomicrographs showing staining for early neural markers (magnification, $40 \times$ ), including SOX2 (red, upper left), SOX3 (red, upper middle), PAX6 (green, upper right), Nestin (green, lower left), Musashi-1 (MS1, red, lower middle), and PSA-NCAM (red, lower right). DAPI in blue. (C) Flow cytometry characterization of representative AF-NPC-SeVs (positive shown in blue compared with red negative control without antibody) at passage 5 demonstrating expression of the neural progenitor cell surface marker PSA-NCAM and lack of staining of the pluripotency marker SSEA4. (D) Representative RT-PCR showing loss of NANOG and OCT4 expression but gain in SOX1 and PAX6 expression after directed differentiation to neural progenitors (AF-NPC$\mathrm{SeV}$ ). (E) Representative karyotype analysis of AF-NPC-SeVs based on $20 \mathrm{G}$-banded metaphase cells demonstrating normal chromosomes (passage 8). (F) Eight days after transplantation of AF-NPC-SeVs into the brain parenchyma of neonatal rats, evidence for engraftment is demonstrated by immunoreactivity to human nuclear antigen (HNA, red) antibodies. Shown are representative merged photomicrographs magnified at $20 \times($ left $), 100 \times($ center $)$, and $100 \times$ confocal (right). DAPI in blue. Color images available online at www.liebertpub.com/scd

aimed at differentiation into both neuronal and glial phenotypes. AF-NPC-SeVs maintained in neurogenic media for up to 2 months were capable of efficient differentiation into neurons. Phase-contrast microscopy showed characteristic morphological changes including cells with extension of neurites as early as 12 days after induction (Fig. 7A, upper left). Immunofluorescence staining revealed robust expression of the early neuronal marker doublecortin (DCX) as well as more mature neuronal markers, including TuJ1 and MAP2 (Fig. 7A). Loss of PAX6 amplification and increased amplification of MAP2 was observed by RT-PCR (data not shown). There was some degree of nonspecific neural dif- ferentiation evidenced by the expression of GFAP but not Olig2 following neuronal induction (data not shown).

To determine whether AF-NPC-SeVs were capable of differentiating toward a glial fate, AF-NPC-SeVs maintained in neural induction media were switched to astrocyte media for 4 weeks. Over the ensuing weeks, the majority of cells adopted a stellate morphology. Robust astrocyte differentiation was evidenced by marked upregulation of GFAP but not Olig2 by RT-PCR (Fig. 7B). Following astrocyte induction, nestin and MSI1 amplification was still expressed but at reduced levels. Evidence for the continued presence of neuronal dendrites was supported by ongoing 


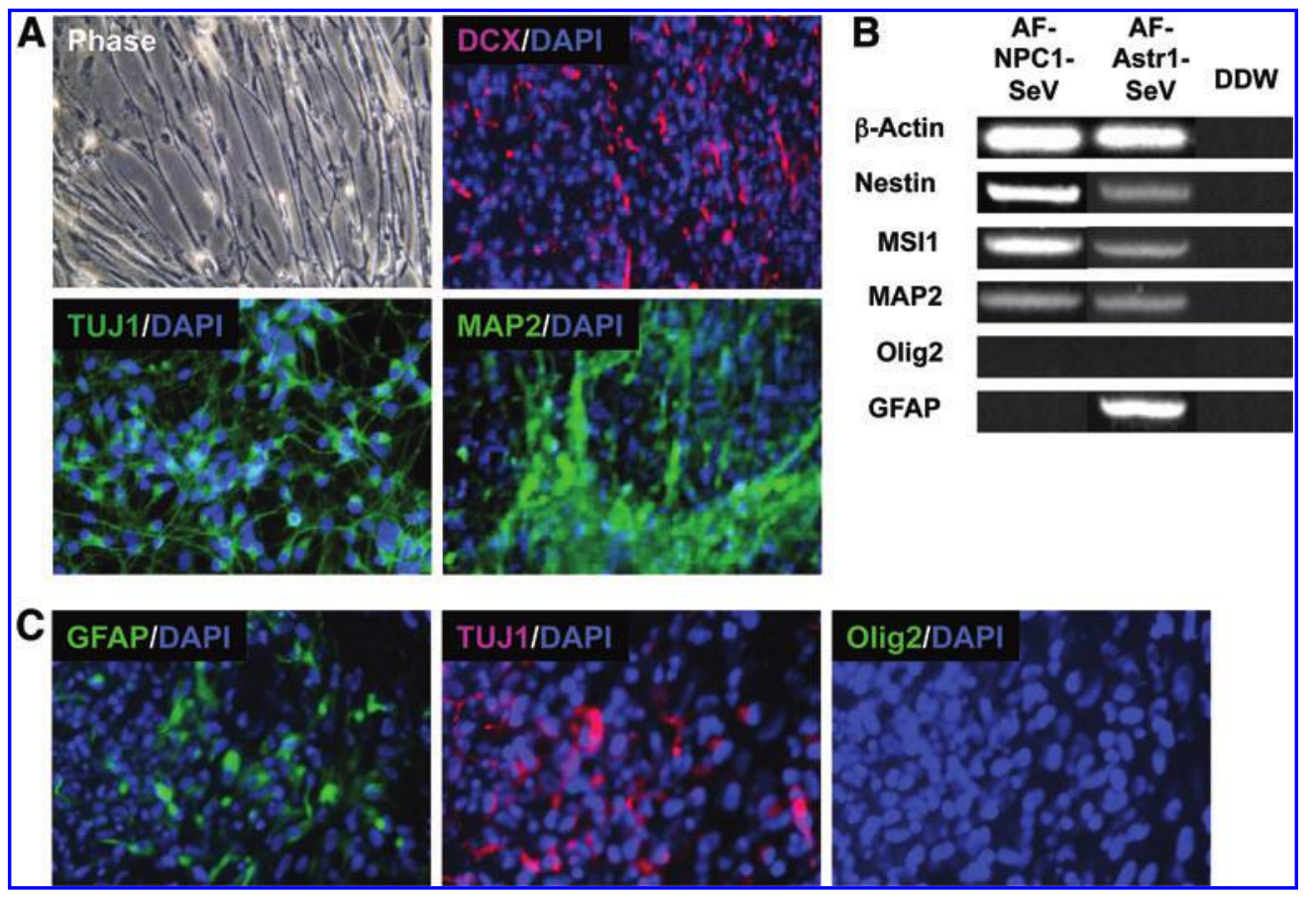

FIG. 7. Characterization of neural progenitors from human amniotic-fluid-derived iPSCs in vitro. (A) Morphology of AF-NPC-SeVs after directed differentiation into the neuronal lineage viewed under phase microscopy at day 12 (upper left, magnification, $100 \times)$. Merged photomicrographs at day 70 showing immunofluorescence for several neuronal markers (magnification, $40 \times$ ), including doublecortin (DCX, purple, upper right), neuron-specific class III $\beta$-tubulin (TuJ1, green, lower left), and microtubule-associated protein-2 (MAP2, green, lower right). DAPI in blue. (B) RT-PCR exhibiting gain in glial fibrillary acid protein (GFAP) expression after directed differentiation of neural progenitors (AFNPC1-Sev) toward the astrocyte (AF-Astr1-SeV) lineage. (C) Merged photomicrographs (magnification, 40 $\times$ ) after directed differentiation of AF-NPC1-SeV toward astrocytes, demonstrating immunofluorescence staining of selected cells for GFAP (left) and TuJ1 (middle) but not Olig2 (right), superimposed with blue DAPI. Color images available online at www.liebertpub.com/scd

transcription of MAP2. Immunofluorescence staining showed the increased presence of GFAP-positive cells that were not normally expressed by AF-NPC-SeVs and more mature neuronal cells. There were scattered, rare areas showing TuJ1-positive cells (Fig. 7C). Olig2 expression was uniformly low, confirming that the glial lineage differentiation of transgene-free neural progenitors was directed toward an astrocytic phenotype.

\section{Discussion}

While postnatal dermal fibroblasts will likely remain the somatic cell of choice for basic research to mechanistically dissect most cellular reprogramming processes, iPSC derivation for therapeutic purposes will ultimately require that the candidate parental population to be readily accessible, easy to reprogram, and free from genetic aberrations [15]. For the fetus with a prenatally diagnosed anomaly amendable to cell transplantation, AF-MSCs would be ideal for facilitating early and effective stem cell therapy. At 28 weeks of gestation, from a mean volume of amniotic fluid of $800 \mathrm{~mL}$, a $10 \mathrm{~mL}$ aliquot of fluid obtained under ultrasound guidance could yield a large starting population of AFMSCs within several weeks. Moreover, an amniocentesis would avoid the inherent risk of fetal injury and/or demise associated with fetal skin biopsy.
In this report, we successfully derive new human transgene-free iPSC lines from AF-MSCs isolated from second- and third-trimester amniotic fluid. The resultant iPSCs were virtually indistinguishable from H9-ESCs in multiple assays and, in support of this therapeutic concept, could be used to generate a relatively homogeneous population of neural progenitors, expressing PAX6, SOX2, SOX3, MSI1, and PSA-NCAM. Further, these neural progenitors were capable of differentiating into mature neurons and astrocytes in vitro and showed transient engraftment potential after implantation in a xenogeneic model. Based on the existing protocols employed in this study, a purified population of neural progenitors could be generated from a small aliquot of amniotic fluid in as little as 8 weeks. The reprogramming efficiency of AF-MSCs with nonintegrating $\mathrm{SeV}$ in our study is comparable to reports using integrative techniques on cells derived from other extraembryonic tissue sources, including placenta, Wharton's jelly, and amniotic membrane $[2,16,17]$.

By definition, the AF-MSCs in this study were adherent to plastic in culture, had defined immunophenotypic characteristics, and possessed limited differentiation capabilities [11]. To our knowledge, only one other report has successfully described iPSC reprogramming of AF-MSCs using nonintegrating techniques. In that study, an excisable cassette based on Cre/LoxP technology was employed to 
generate iPSCs from a fetus with thalassemia [18]. Although this technique is relatively efficient, an $\sim 200$-bp fragment of inactive viral LTR remains in the host genome after an inherently labor-intensive process involving the identification of clones with vector integration. Further, directed differentiation of integration-free iPSCs derived from amniocytes into a homogeneous and clinically relevant cell type has not been reported.

More recently, Guillot and colleagues elegantly demonstrated a transgene-free approach employing the histone deacetylase inhibitor valproic acid to induce pluripotency from amniotic fluid cells [19]. A key distinction in this study, however, is the use of CD117-positive AFS cells, a clonal population of somatic cells that requires magnetic bead sorting to identify these rare $(<1 \%)$ amniocytes, followed by months of in vitro culture. Hierarchical clustering data showed that the valproic-acid-exposed AFS cells are more closely related to native AFS cells than to H9-ESCs. Further, the use of AFS-cell-based therapies for autologous purposes may be difficult since it remains unclear whether these CD117-positive cells are present in every specimen, and particularly in those beyond 22 weeks of gestation, the time period when many prenatal anomalies are initially characterized by maternal-fetal medicine clinicians with ultrasound imaging $[20,21]$. By the third trimester of pregnancy, the pluripotency of fetal cells derived from other extraembryonic tissues also seems to become more restricted [1].

Purified populations of neural cells derived from transgenefree AF-iPSCs in vitro may facilitate the development of tissue engineering and other cell-based therapies for some of the most difficult perinatal disorders treated in neonatal intensive care units worldwide [22]. Specific neurological diseases, all of which are manifested by damage or loss of organ function, would include neonatal hypoxic-ischemic encephalopathy, cerebral palsy, myelomeningocele, and structural brain malformations. In our study, neural progenitors were generated from one recipient fetus with TTTS, a condition associated with a sevenfold increased risk of long-term neurologic morbidity secondary to antenatal cerebral hypoperfusion in the donor twin with hyperviscosity and polycythemia in the recipient twin [23]. Other neonates who might also benefit from AF-iPSC-based therapies include those with significant nongenetic prenatal abnormalities involving the heart, lungs, and liver.

The use of AF-MSCs as a substrate for transgene-free iPSC reprogramming offers many other potential advantages in regenerative medicine beyond the perinatal period. Recent work has shown that reprogrammed cells at early passages have varied differentiation potential and residual DNA methylation signatures that are biased toward their original cell phenotype, commonly referred to as tissuespecific "epigenetic memory" [24,25]. It would therefore be ideal to use somatic cells with an immature ground state, such as those originating from fetal-derived tissues, to efficiently generate fully competent iPSCs amendable for subsequent differentiation into the target cell of choice. Because of the embryonic/fetal origins of AF-MSCs, these cells are believed to have an intermediate phenotype between that of true pluripotent stem cells and adult somatic stromal cells [26,27].

Whereas the delivery of autologous cells based on AFiPSCs might be appealing and may prove more practical for certain regenerative medicine applications, allogeneic transplantation using cryopreserved cells derived from human leukocyte antigen (HLA)-matched AF-iPSCs stored within good manufacturing practice biorepositories represents another viable alternative. An allogeneic approach would conceivably offer some additional advantages, enabling the use of HLA-matched cells "off-the-shelf" without the need for immunosuppression, might also be feasible in this setting given the known immunoregulatory properties of amnion-derived MSCs within the host environment [27-29].

Our finding of the increased "stemness" of AF-MSCs based on pluripotency markers parallels the work of others and suggests similar transcriptional and epigenetic states compared with ESCs as well as enhanced reprogramming capabilities [30-33]. Evidence to further support these concepts include maintenance of long, stable telomeres in culture and high reprogramming efficiencies among iPSCs created using integrating ectopic factors. Recent data also suggest that these cells have a complex molecular signature, coexpressing trophoblastic, ectodermal, mesodermal, and endodermal cell-type-specific regulators [34]. All of these characteristics lend support to the potential benefits of banking amniotic fluid for future, patient-specific therapeutic applications later in childhood and adult life.

Because of the relatively high expression of selected pluripotency markers among AF-MSCs, there may be a unique subset of cells within a heterogeneous population that have even better reprogramming efficiency using $\mathrm{SeV}$ or other nonintegrating techniques. The identification of certain amniocyte subpopulations with enhanced reprogramming ability may also allow direct reprogramming into other cell types as was recently demonstrated [35]. The use of fewer Yamanaka reprogramming transgenes to achieve pluripotency within human amniocytes has also been shown [36]. Our work will likely stimulate further research comparing iPSCs derived from different human fetal sources in an effort to better understand the phenomenon of pluripotency and tissue-specific epigenetic memory, both in vitro and in vivo.

While we did not formally compare AF-MSC characteristics based on the presence or absence of a fetal anomaly, we speculate that any differences in reprogramming characteristics or neural progenitor differentiation based on prenatal diagnosis are likely to be minimal. In this study, we also did not attempt to differentiate the AF-MSCs into various germ layer derivatives in the absence of reprogramming with $\mathrm{SeV}$. Consistent with findings from another study [29], our data on somatic cells derived from amniotic tissues suggest that they have a restricted differentiation potential at baseline, as shown by minimal osteogenic and chondrogenic behavior when compared with bone-marrowderived MSCs. The general consensus based on our past experience, among others, has been that AF-MSCs are not fully pluripotent since they do not generally form EBs in low-attachment dishes. As a result, we could not readily differentiate these cells toward the neural lineage based on existing protocols. Some groups have shown upregulation of neural-specific genes among AF-MSCs under specific culture conditions [37], but compelling evidence for the differentiation of neural progenitors from truly multipotent stem cells as opposed to committed precursor cells has not been clearly demonstrated [38]. 
In conclusion, the present study demonstrates that human AF-MSCs from a wide range of gestational ages and prenatal conditions can be reliably isolated and induced into iPSCs and neural progenitors free of reprogramming factors using $\mathrm{SeV}$. Perinatal animal experiments in the laboratory that utilizes the derivatives of these iPSCs remain ongoing and will hopefully facilitate the eventual development of therapeutic-grade iPSCs for fetuses and young children with debilitating diseases.

\section{Acknowledgments}

This work was supported by the Robert Wood Johnson Foundation (Harold Amos Scholarship to Dr. Kunisaki) and the American College of Surgeons (James Carrico Award to Dr. Kunisaki). The authors thank Jeannie Kreutzman and Dr. Kristie L. Keeton for facilitating the collection of specimens, and Joseph Washburn and Craig Johnson for assistance with the microarray analysis.

Presented in part at the 2013 American Pediatric Surgical Association Annual Meeting, Marco Island, Florida.

\section{Author Disclosure Statement}

No competing financial interests exist for each author on this article.

\section{References}

1. Jones GN, D Moschidou, TI Puga-Iglesias, K Kuleszewicz, M Vanleene, SJ Shefelbine, Bou-Gharios G, NM Fisk, AL David, De Coppi P and Guillot PV. (2012). Ontological differences in first compared to third trimester human fetal placental chorionic stem cells. PLoS One 7:e43395.

2. Cai J, W Li, H Su, D Qin, J Yang, F Zhu, J Xu, W He, X Guo, et al. (2010). Generation of human induced pluripotent stem cells from umbilical cord matrix and amniotic membrane mesenchymal cells. J Biol Chem 285:11227-11234.

3. Fujioka T, N Shimizu, K Yoshino, H Miyoshi and Y Nakamura. (2010). Establishment of induced pluripotent stem cells from human neonatal tissues. Hum Cell 23:113-118.

4. Lu HE, YC Yang, SM Chen, HL Su, PC Huang, MS Tsai, TH Wang, CP Tseng and SM Hwang. (2013). Modeling neurogenesis impairment in Down syndrome with induced pluripotent stem cells from Trisomy 21 amniotic fluid cells. Exp Cell Res 319:498-505.

5. Miki T, T Lehmann, H Cai, DB Stolz and SC Strom. (2005). Stem cell characteristics of amniotic epithelial cells. Stem Cells 23:1549-1559.

6. Kunisaki SM, M Armant, GS Kao, K Stevenson, H Kim and DO Fauza. (2007). Tissue engineering from human mesenchymal amniocytes: a prelude to clinical trials. J Pediatr Surg 42:974-979; discussion 979-980.

7. Di Cagno A, C Baldari, C Battaglia, P Brasili, F Merni, M Piazza, S Toselli, AR Ventrella and L Guidetti. (2008). Leaping ability and body composition in rhythmic gymnasts for talent identification. J Sports Med Phys Fitness 48:341-346.

8. Villa-Diaz LG, SE Brown, Y Liu, AM Ross, J Lahann, JM Parent and PH Krebsbach. (2012). Derivation of mesenchymal stem cells from human induced pluripotent stem cells cultured on synthetic substrates. Stem Cells 30:1174-1181.

9. Villa-Diaz LG, H Nandivada, J Ding, NC Nogueirade-Souza, PH Krebsbach, KS O'Shea, J Lahann and GD
Smith. (2010). Synthetic polymer coatings for long-term growth of human embryonic stem cells. Nat Biotechnol 28:581-583.

10. Mak SK, YA Huang, S Iranmanesh, M Vangipuram, R Sundararajan, L Nguyen, JW Langston and B Schule. (2012). Small molecules greatly improve conversion of human-induced pluripotent stem cells to the neuronal lineage. Stem Cells Int 2012:140427.

11. Dominici M, K Le Blanc, I Mueller, I Slaper-Cortenbach, F Marini, D Krause, R Deans, A Keating, D Prockop and E Horwitz. (2006). Minimal criteria for defining multipotent mesenchymal stromal cells. The International Society for Cellular Therapy position statement. Cytotherapy 8:315317.

12. Bentley JK, AP Popova, PD Bozyk, MJ Linn, AE Baek, J Lei, AM Goldsmith and MB Hershenson. (2010). Ovalbumin sensitization and challenge increases the number of lung cells possessing a mesenchymal stromal cell phenotype. Respir Res 11:127.

13. Itskovitz-Eldor J, M Schuldiner, D Karsenti, A Eden, O Yanuka, M Amit, H Soreq and N Benvenisty. (2000). Differentiation of human embryonic stem cells into embryoid bodies compromising the three embryonic germ layers. Mol Med 6:88-95.

14. Livak KJ and TD Schmittgen. (2001). Analysis of relative gene expression data using real-time quantitative PCR and the 2(-Delta Delta C(T)) Method. Methods 25:402-408.

15. Maherali N and K Hochedlinger. (2008). Guidelines and techniques for the generation of induced pluripotent stem cells. Cell Stem Cell 3:595-605.

16. Li C, J Zhou, G Shi, Y Ma, Y Yang, J Gu, H Yu, S Jin, Z Wei, F Chen and Y Jin. (2009). Pluripotency can be rapidly and efficiently induced in human amniotic fluid-derived cells. Hum Mol Genet 18:4340-4349.

17. Lu HE, MS Tsai, YC Yang, CC Yuan, TH Wang, XZ Lin, CP Tseng and SM Hwang. (2011). Selection of alkaline phosphatase-positive induced pluripotent stem cells from human amniotic fluid-derived cells by feeder-free system. Exp Cell Res 317:1895-1903.

18. Fan Y, Y Luo, X Chen, Q Li and X Sun. (2012). Generation of human beta-thalassemia induced pluripotent stem cells from amniotic fluid cells using a single excisable lentiviral stem cell cassette. J Reprod Dev 58:404-409.

19. Moschidou D, S Mukherjee, MP Blundell, K Drews, GN Jones, H Abdulrazzak, B Nowakowska, A Phoolchund, K Lay, et al. (2012). Valproic acid confers functional pluripotency to human amniotic fluid stem cells in a transgenefree approach. Mol Ther 20:1953-1967.

20. Moschidou D, S Mukherjee, MP Blundell, GN Jones, AJ Atala, AJ Thrasher, NM Fisk, P De Coppi and PV Guillot. (2013). Human mid-trimester amniotic fluid stem cells cultured under embryonic stem cell conditions with valproic Acid acquire pluripotent characteristics. Stem Cells Dev 22:444-458.

21. Machtay M, R Paulus, J Moughan, R Komaki, J Bradley, H Choy, K Albain, B Movsas, WT Sause and WJ Curran. (2012). Defining local-regional control and its importance in locally advanced non-small cell lung carcinoma: a radiation therapy oncology group analysis. J Thorac Oncol 7:716-722.

22. Fauza D. (2004). Amniotic fluid and placental stem cells. Best Pract Res Clin Obstet Gynaecol 18:877-891.

23. Simpson LL. (2013). Twin-twin transfusion syndrome. Am J Obstet Gynecol 208:3-18. 
24. Hui L, DK Slonim, HC Wick, KL Johnson and DW Bianchi. (2012). The amniotic fluid transcriptome: a source of novel information about human fetal development. $\underline{\mathrm{Ob}-}$ stet Gynecol 119:111-118.

25. Polo JM, S Liu, ME Figueroa, W Kulalert, S Eminli, KY Tan, E Apostolou, M Stadtfeld, Y Li, et al. (2010). Cell type of origin influences the molecular and functional properties of mouse induced pluripotent stem cells. Nat Biotechnol 28:848-855.

26. Evangelista M, M Soncini and O Parolini. (2008). Placentaderived stem cells: new hope for cell therapy? Cytotechnology 58:33-42.

27. Lee JM, J Jung, HJ Lee, SJ Jeong, KJ Cho, SG Hwang and GJ Kim. (2012). Comparison of immunomodulatory effects of placenta mesenchymal stem cells with bone marrow and adipose mesenchymal stem cells. Int Immunopharmacol 13:219-224.

28. Bailo M, M Soncini, E Vertua, PB Signoroni, S Sanzone, G Lombardi, D Arienti, F Calamani, D Zatti, et al. (2004). Engraftment potential of human amnion and chorion cells derived from term placenta. Transplantation 78:1439-1448.

29. Wegmeyer H, AM Broske, M Leddin, K Kuentzer, AK Nisslbeck, J Hupfeld, K Wiechmann, J Kuhlen, C von Schwerin, et al. (2013). Mesenchymal stromal cell characteristics vary depending on their origin. Stem Cells Dev 22:2606-2618.

30. Guillot PV, C Gotherstrom, J Chan, H Kurata and NM Fisk. (2007). Human first-trimester fetal MSC express pluripotency markers and grow faster and have longer telomeres than adult MSC. Stem Cells 25:646-654.

31. Anchan RM, P Quaas, B Gerami-Naini, H Bartake, A Griffin, Y Zhou, D Day, JL Eaton, LL George, et al. (2011). Amniocytes can serve a dual function as a source of iPS cells and feeder layers. Hum Mol Genet 20:962-974.

32. Koo BK, IY Park, J Kim, JH Kim, A Kwon, M Kim, Y Kim, JC Shin and JH Kim. (2012). Isolation and characterization of chorionic mesenchymal stromal cells from human full term placenta. J Korean Med Sci 27:857-863.

33. Fariha MM, KH Chua, GC Tan, AE Tan and AR Hayati. (2011). Human chorion-derived stem cells: changes in stem cell properties during serial passage. Cytotherapy 13: 582-593.

34. Maguire CT, BL Demarest, JT Hill, JD Palmer, AR Brothman, HJ Yost and ML Condic. (2013). Genome-wide analysis reveals the unique stem cell identity of human amniocytes. PLoS One 8:e53372.

35. Ginsberg M, D James, BS Ding, D Nolan, F Geng, JM Butler, W Schachterle, VR Pulijaal, S Mathew, et al. (2012). Efficient direct reprogramming of mature amniotic cells into endothelial cells by ETS factors and TGFbeta suppression. Cell 151:559-575.

36. Liu T, G Zou, Y Gao, X Zhao, H Wang, Q Huang, L Jiang, L Guo and W Cheng. (2012). High efficiency of reprogramming $\mathrm{CD} 34(+)$ cells derived from human amniotic fluid into induced pluripotent stem cells with Oct4. Stem Cells Dev 21:2322-2332.

37. Macias MI, J Grande, A Moreno, I Dominguez, R Bornstein and AI Flores. (2010). Isolation and characterization of true mesenchymal stem cells derived from human term decidua capable of multilineage differentiation into all 3 embryonic layers. Am J Obstet Gynecol 203:495 e9-495 e23.

38. Toselli M, E Cerbai, F Rossi and E Cattaneo. (2008). Do amniotic fluid-derived stem cells differentiate into neurons in vitro? Nat Biotechnol 26:269-270; Author Reply 270-261.

Address correspondence to: Shaun M. Kunisaki, MD, MSc Department of Surgery C.S. Mott Children's Hospital and Von Voigtlander Women's Hospital University of Michigan 1540 East Hospital Drive, SPC 4211 Ann Arbor, MI 48109

E-mail:shaunkun@umich.edu

Received for publication February 28, 2014 Accepted after revision July 11, 2014

Prepublished on Liebert Instant Online July 11, 2014 


\section{This article has been cited by:}

1. Kehl Debora, Generali Melanie, Görtz Sabrina, Geering Diego, Slamecka Jaroslav, Hoerstrup Simon P., Bleul Ulrich, Weber Benedikt. 2017. Amniotic Fluid Cells Show Higher Pluripotency-Related Gene Expression Than Allantoic Fluid Cells. Stem Cells and Development 26:19, 1424-1437. [Abstract] [Full Text HTML] [Full Text PDF] [Full Text PDF with Links]

2. Aaron J. Velasquez-Mao, Christopher J. M. Tsao, Madeline N. Monroe, Xavier Legras, Beatrice Bissig-Choisat, Karl-Dimiter Bissig, Rodrigo Ruano, Jeffrey G. Jacot. 2017. Differentiation of spontaneously contracting cardiomyocytes from non-virally reprogrammed human amniotic fluid stem cells. PLOS ONE 12:5, e0177824. [Crossref]

3. Stefano Da Sacco, Astgik Petrosyan, Laura Perin. Amniotic Fluid Cells 1097-1107. [Crossref]

4. Vardine Sahakyan, Enrico Pozzo, Robin Duelen, Jan Deprest, Maurilio Sampaolesi. 2017. Methotrexate and Valproic Acid Affect Early Neurogenesis of Human Amniotic Fluid Stem Cells from Myelomeningocele. Stem Cells International 2017, 1-10. [Crossref]

5. Jake Sokol, Trenton Lippert, Cesar V. Borlongan, Liborio Stuppia. 2016. Translating amniotic fluid-derived stem cells for transplantation in stroke. Chinese Neurosurgical Journal 2:1. . [Crossref]

6. Guihua Jiang, Todd J. Herron, Julie Di Bernardo, Kendal A. Walker, K. Sue O’Shea, Shaun M. Kunisaki. 2016. Human Cardiomyocytes Prior to Birth by Integration-Free Reprogramming of Amniotic Fluid Cells. STEM CELLS Translational Medicine 5:12, 1595-1606. [Crossref]

7. Dziadosz Margaret, Chan Michael, Basch Ross, Young Bruce K.. 2016. Effects of Pharmacological Agents on Human Amniotic Fluid-Derived Stem Cells in Culture. Stem Cells and Development 25:20, 1570-1579. [Abstract] [Full Text HTML] [Full Text PDF] [Full Text PDF with Links]

8. Ivana Antonucci, Martina Provenzano, Melissa Rodrigues, Andrea Pantalone, Vincenzo Salini, Patrizia Ballerini, Cesar Borlongan, Liborio Stuppia. 2016. Amniotic Fluid Stem Cells: A Novel Source for Modeling of Human Genetic Diseases. International Journal of Molecular Sciences 17:4, 607. [Crossref]

9. Margaret Dziadosz, Ross S. Basch, Bruce K. Young. 2016. Human amniotic fluid: a source of stem cells for possible therapeutic use. American Journal of Obstetrics and Gynecology 214:3, 321-327. [Crossref]

10. Jaroslav Slamecka, Lilia Salimova, Steven McClellan, Mathieu van Kelle, Debora Kehl, Javier Laurini, Paolo Cinelli, Laurie Owen, Simon P Hoerstrup, Benedikt Weber. 2016. Non-integrating episomal plasmid-based reprogramming of human amniotic fluid stem cells into induced pluripotent stem cells in chemically defined conditions. Cell Cycle 15:2, 234-249. [Crossref]

11. Chang-qing Jiang, Jun Hu, Jian-ping Xiang, Jia-kai Zhu, Xiao-lin Liu, Peng Luo. 2016. Tissue-engineered rhesus monkey nerve grafts for the repair of long ulnar nerve defects: similar outcomes to autologous nerve grafts. Neural Regeneration Research 11:11, 1845. [Crossref]

12. Kai-Hung Wang, An-Pei Kao, Chia-Cheng Chang, Ta-Chin Lin, Tsung-Cheng Kuo. 2015. Upregulation of Nanog and Sox-2 genes following ectopic expression of Oct-4 in amniotic fluid mesenchymal stem cells. Biotechnology and Applied Biochemistry 62:5, 591-597. [Crossref]

13. Maya Elias, Jaclyn Hoover, Hung Nguyen, Stephanny Reyes, Christopher Lawton, Cesar V Borlongan. 2015. Stroke therapy: the potential of amniotic fluid-derived stem cells. Future Neurology 10:4, 321-326. [Crossref]

14. Patricia G. Wilson, Tiffany Payne. 2014. Genetic reprogramming of human amniotic cells with episomal vectors: neural rosettes as sentinels in candidate selection for validation assays. PeerJ 2, e668. [Crossref] 